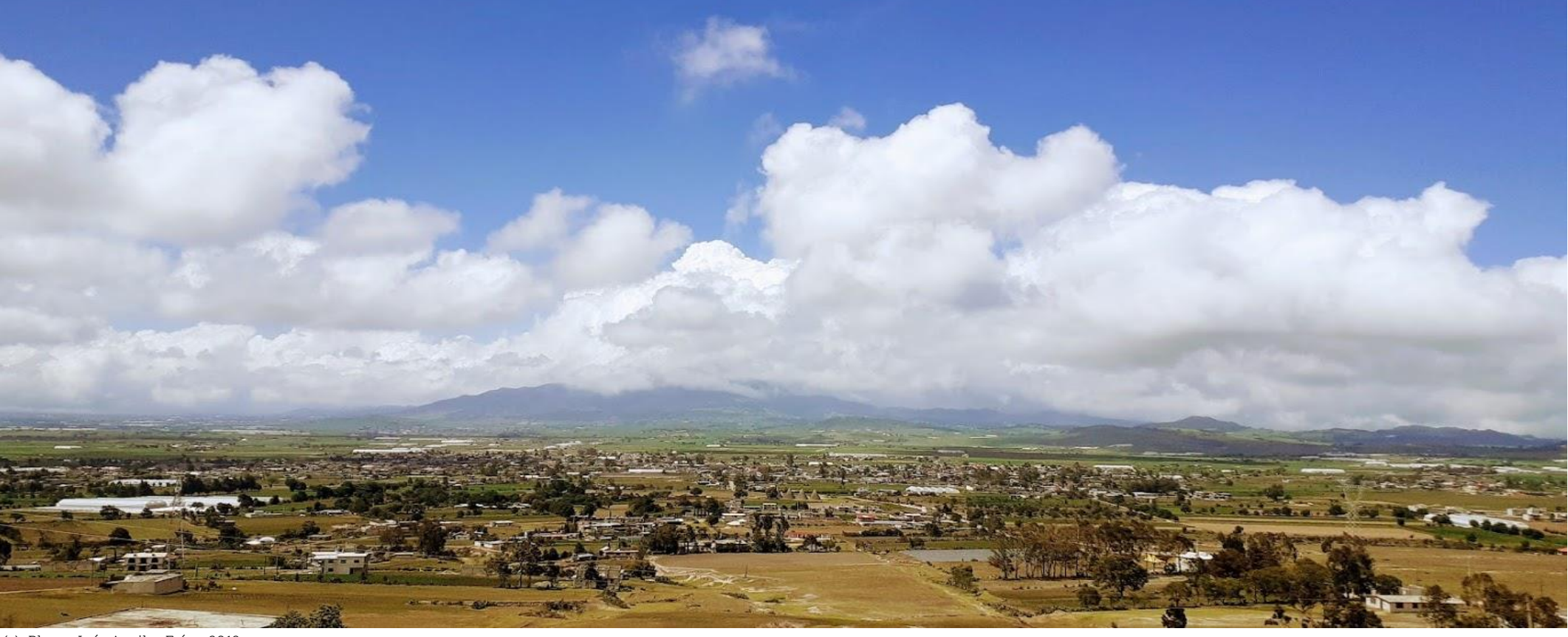

(c) Blanca Inés Aguilar Frías. 2019

\title{
Movimientos sociales, conservación del paisaje biocultural y ecoturismo solidario en el corredor Mazunte-Escobilla, Oaxaca, México
}

\author{
Diana Areli Zárate Ángela - Rubén Cantú Chapab - José Teodoro Silva Garcíac - Yoana \\ Hernández Suárez ${ }^{d}$
}

RESUMEN: Los ecoturismos que acontecen en el corredor Mazunte-Escobilla, Oaxaca, México han posibilitado la conservación de sus paisajes bioculturales. Esta actividad, ha sido una opción para cooperativas que llevan a cabo actividades de economía solidaria y comercio justo, resultado de una movilización comunitaria por la defensa del territorio como espacio

\footnotetext{
a Candidata a Doctora en Ciencias en Conservación del Patrimonio Paisajístico. Adscrita al Centro Interdisciplinario de Investigaciones y Estudios sobre Medio Ambiente y Desarrollo, Instituto Politécnico Nacional. ORCID: https://orcid.org/00000003-4081-8379. Contacto: dangel.ciiemad@gmail.com

b Doctorado en Urbanismo, Unidad de Posgrado de la Facultad de Arquitectura-Universidad Nacional Autónoma de México. Contacto: rcantuch@yahoo.com.

c Centro Interdisciplinario de Investigación para el Desarrollo Integral Regional, Unidad Michoacán del Instituto Politécnico Nacional. Contacto: tsilva09@hotmail.com.

d Doctora en Ciencias Históricas por la Universidad de La Habana. Investigadora titular el Instituto de Historia de Cuba y profesora adjunta de la Universidad de La Habana. Contacto: yohs1972@gmail.com.
} 
de apropiación frente a la prohibición de una de las actividades económicas más importantes de la región: la caza de tortuga. En este sentido, se cuestionó si el ecoturismo puede propiciar un escenario de conservación de los paisajes bioculturales en el largo plazo. Para responder dicha aseveración, se utilizó una metodología cualitativa basada en entrevistas semiestructuradas dirigidas a actores estratégicos mediante un análisis inferencial opinático, el cual se contrastó con la revisión del Estado del Arte del territorio. Algunos hallazgos muestran una correlación entre la recomposición de los vínculos en la comunidad gracias a los ecoturismos resultado de la movilización social.

Palabras clave: Movimientos Sociales; Ecoturismo; Paisajes Bioculturales; Economía Solidaria; Conservación.

\begin{abstract}
There are some kinds of ecotourisms that occur in the Mazunte-Escobilla corridor, Oaxaca, Mexico which have enabled the conservation of their biocultural landscapes. Firstly, this activity has been an option for cooperatives that carry out solidarity economy and fairtrade activities, as a result of a community mobilization for the defense of the territory as a space of appropriation against the prohibition of one of the most important economic activities in the region: turtle hunting. In this regard, it was questioned whether ecotourism could be a scenario of conservation of biocultural landscapes in the long term. Qualitative methodology was used based on semi-structured interviews aimed at strategic actors through an inferential opinion analysis, which it was contrasted with the revision of the State of the Art of the territory. Finally, some findings show a correlation between the recomposition of the bonds in the community thanks to the ecotourisms resulting from social mobilization.
\end{abstract}

Keywords: Social Movements; Ecotourism; Biocultural; Landscapes; Solidarity Economy; Conservation.

\title{
Introducción
}

$\mathrm{U}$

n paisaje biocultural es "un territorio que comparte un paisaje e identidad propia, manejado bajo un régimen unificado de gestión territorial que permite promover el desarrollo económico sustentable por medio de la protección y valoración de la naturaleza y la cultura local" (Bezaury-Creel et al., 2015, p. 30). Por su parte, Boege (2008 cit. en Luque et. al. 2018) sostiene que la vertiente biocultural se distingue por considerar la cultura como una mediación entre la sociedad y la naturaleza que determina la manera en 
Sección Especial - Territorios, Sustentabilidad, Cultura y Transformación de Espacios

que se realiza y se recrea la subsistencia humana. De esta forma, la dimensión cultural tiene una relevancia particular, en la que se detonan ciertos aspectos de la cosmovisión, de las prácticas productivas y de los saberes relacionados con el aprovechamiento de la biodiversidad (p. 16).

Para fines de la presente investigación, un paisaje biocultural se caracteriza por la autonomía comunitaria en donde las formas de usos y costumbres ponen un valor al territorio. Además, es propulsor de iniciativas de economía solidaria y comercio justo dadas la visión comunitaria en tanto al valor inmaterial de los territorios, por lo que se propulsa el reconocimiento de los derechos políticos, culturales y ambientales de las comunidades que lo habitan (Zarate, 2019).

Reconsiderar al territorio desde una visión de respeto por los paisajes bioculturales requiere, entonces, de una labor de voluntad, no solo comunitaria, sino también política para resarcir los conflictos que en los paisajes del corredor Mazunte-Escobilla, Oaxaca, México, persisten; motivo por el cual su estudio representó dos retos metodológicos: por un lado, el análisis etnográfico del territorio que, de acuerdo con Cefaï (2013, p. 101), es un proceso investigativo que se sustenta en una observación prolongada, continua o fraccionada, de situaciones, en espacios públicos, organizaciones o comunidades.

Por el otro, el conflicto socio ambiental de lucha que ha enfrentado el territorio es una relación compleja de variables sociales, económicas y ambientales que permiten apreciar matices diversos de la problemática territorial que de cierta forma fueron subsanadas de fondo por diversas organizaciones cooperativas que apostaron a una nueva forma de verse y reconocer la importancia de su comunicación con las y los otros. Esta forma decantó en un ecoturismo solidario.

En este sentido, el objetivo de la presente investigación fue determinar qué tipo de ecoturismo puede propiciar un escenario de conservación de los paisajes bioculturales en el largo plazo, pues en la actualidad se presentan diversos modelos de economía (desde la economía tradicional hasta la economía solidaria), a través de actividades denominadas "ecoturísticas", que pudiesen poner en riesgo la permanencia de estos espacios excepcionales. Luego entonces, sin ser un estudio exhaustivo.

A continuación, se muestran los hallazgos obtenidos durante la primera etapa de la investigación desde un análisis descriptivo correlacional donde se generó un breve análisis histórico de la vida comunitaria de finales de siglo xx en las comunidades de Mazunte (principalmente por ser el eje histórico como se verá más adelante), Ventanilla y Escobilla. 


\section{Sección Especial - Territorio, Sustentabilidad, Cultura y Transformación de Espacios}

Ésta primera etapa, permitió identificar cómo un movimiento social comunitario decantó en negociaciones con diversos actores políticos que beneficiaron a las comunidades del corredor Mazunte-Escobilla a través del ecoturismo, además de observar ciertos aspectos que pueden poner en riesgo la conservación de los paisajes bioculturales del área de estudio.

\section{Sustentabilidad, sociedad de consumo y ecoturismo}

Bauman (1998, p. 106) sostiene que, "nuestra sociedad, es una sociedad de consumo" y, por ende, los procesos globalizadores incluyen una segregación, separación y marginación social progresiva. Frente a escenarios inciertos en tanto a los derechos de la vida comunitaria y de los pueblos originarios de Latinoamérica, conceptos relacionados a la sustentabilidad intentan armonizar la lógica del desarrollo capitalista con la conservación ambiental. Por ejemplo, Baumhackl (2003, p. 7) sugiere que, el concepto de sustentabilidad "está ligado al desarrollo de la comunidad y al derecho de las generaciones en el futuro, lo cual obliga a evaluar las acciones humanas en una perspectiva a largo plazo." Y agrega que: "El desarrollo pleno de las generaciones futuras demanda de la generación actual la protección total del medio ambiente (aire, agua, tierra, biodiversidad) y el manejo cuidadoso de los recursos naturales".

Quintana (2004 cit. en Pérez, 2017), menciona que los conflictos ambientales son problemas de carácter político (p. 67). Por su parte Pérez (2017, p. 64) advierte que los conflictos ambientales van más allá de la apropiación de los recursos, para orientarse a la defensa de formas de vida y cultura de pueblos originarios. Barrera-Bassols y Toledo (2018) sostienen que, durante los últimos años se han detectado unos 560 conflictos socio ambientales en el país, muchos de ellos de devastación biocultural directa y otros más con efectos indirectos o secundarios. Siendo el Estado de Oaxaca quien registra la mayor cantidad de conflictos ambientales dentro de su territorio y que a su vez es la entidad con mayor diversidad biocultural, lo cual muestra la dimensión de este tipo de devastación en el país y la ferviente necesidad de retomar en la discusión política el alcance del disfrute de la naturaleza y el reconocimiento de nuevas formas de entender, gestionar y conservar los territorios manejados desde las comunidades (p. 105).

En contraste con la dinámica desarrollista del planeta y luego de la llamada Revolución Industrial, nace en el mundo una de las actividades económicas que se propulsa como una alternativa de desarrollo entre las comunidades: el turismo. De acuerdo con la Coordinación de Asesores de la Secretaría de Turismo (2018), la llamada "industria sin humo", aporta en la actualidad, según estimaciones de la Organización Mundial del Turismo (OMT), el 10\% del PIB 
Sección Especial - Territorios, Sustentabilidad, Cultura y Transformación de Espacios

Mundial, representa el 30\% de las exportaciones de servicios, genera uno de cada diez empleos y su volumen de negocio, iguala o supera, al de sectores clave como: exportaciones de petróleo, productos alimentarios o automóviles (p. 15).

El turismo, en sus diversas vertientes, ha sido la apuesta para el desarrollo económico de las naciones, sobre todo aquellas actividades de bajo impacto (como el ecoturismo) que se proponen como una nueva forma de vivir experiencias en contacto con las comunidades originarias y la naturaleza. Al respecto, Obombo y Velarde (2019) analizan la relación entre los beneficios y las actitudes de las comunidades locales hacia la conservación en las ANP, encontrándose hallazgos como el hecho de que el ecoturismo puede generar importantes ventajas económicas para las comunidades locales e incentivar su participación en iniciativas de conservación, aunque estos incentivos por sí solos no son suficientes para transformar las actitudes y prácticas de los residentes a favor de la conservación.

Sin embargo, en el imaginario denominado ecoturismo, se presentan diversos niveles de realidad al interior de las comunidades que lo practican. Por un lado, se promueve la idea de lo eco-friendly, donde se genera una especie de ecoaldea que, siguiendo a Wang et al., (2015 cit. en Mardones y Zunino, 2019), responden principalmente a la preocupación por el desarrollo sustentable y la promoción de valores, conductas y prácticas proambientales (p. 4). Por el otro, se genera la apropiación del valor agregado de la actividad y se "vende" en una lógica no tan propiamente comunitaria, experiencias falsas que ponen en riesgo el prestigio del destino y a las propias cooperativas y núcleos comunitarios que verdaderamente practican y propulsan esta actividad. Dicho lo anterior, la figura 1 muestra los ecoturismos existentes en el corredor Mazunte-Escobilla, Oaxaca, México. 


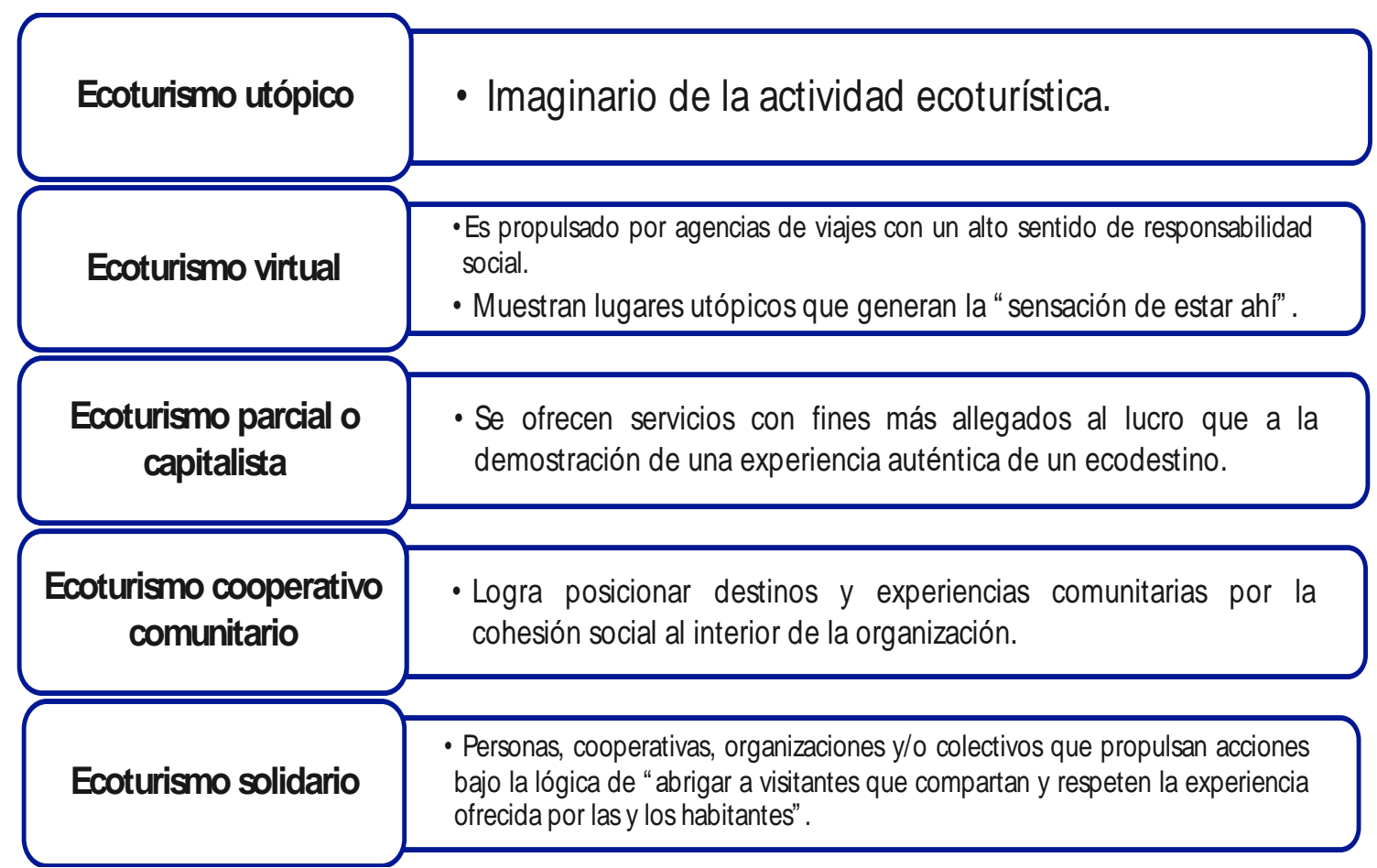

Figura 1. Ecoturismos en el Corredor Mazunte-Escobilla, Oaxaca, México.

Fuente: Elaboración propia

\section{Materiales y métodos}

La investigación realizada fue de corte cualitativo de tipo correlacional con un diseño no experimental transversal, bajo un enfoque etnográfico dividido en tres etapas. Este tipo de estudios han sido utilizados en investigaciones como las de Ortiz (2018) y Rojas \& Palafox (2018) quienes entrecruzaron variables desde el desarrollo comunitario, las Áreas Naturales protegidas y los pueblos mágicos.

La primera etapa, consistió en un análisis histórico que caracterizó el área de estudio y permitió reconocer el Estado del Arte del territorio. Siguiendo el planeamiento de metasíntesis, se revisaron bases de datos, revistas indexadas, artículos científicos y trabajos de investigación nacionales e internacionales que tuviesen injerencia en el área de estudio. El resultado de este cotejo permitió reconocer 42 documentos de trabajo (16 de las ciencias sociales, 17 en materia de ciencias ambientales y 9 de corte interdisciplinario), entre herramientas de planeación, artículos científicos y tesis de diversos grados académicos respecto al territorio de estudio. 
Sección Especial - Territorios, Sustentabilidad, Cultura y Transformación de Espacios

Con base en la información obtenida en esta primera etapa, se prosiguió al desarrollo de un segundo método: el Análisis Inferencial Opinático que contrastó cinco testimonios que se centraron únicamente en la construcción de la memoria histórica-colectiva respecto a la movilización efectuada por la comunidad una vez decretada la veda de la tortuga y que trajo como consecuencia la inserción de nuevos proyectos económicos en el territorio tales como el Centro Mexicano de la Tortuga y las actividades derivadas del ecoturismo. Finalmente, se identificaron diversos tipos de ecoturismo existentes en el territorio de estudio, con lo cual se generaron algunas reflexiones para la conservación de sus paisajes bioculturales.

\section{Localización del área de estudio}

El corredor Mazunte-Escobilla (MAZ-ES, conformado por tres territorios: Mazunte, Ventanilla y el Área Natural Protegida (ANP) Playa de Escobilla), se localiza en el municipio de Santa María Tonameca, perteneciente a la Región Costa del Estado de Oaxaca, México (Ver Figura 2). Mazunte, es una localidad ubicada a 22 kilómetros del Distrito de San Pedro Pochutla, con una población aproximada a las 600 personas, de las cuales, alrededor del 30\% son extranjeros ${ }^{1}$.La comunidad de Ventanilla es una comunidad de aproximadamente 210 personas ${ }^{2}$ que se ubica a 5 minutos en automóvil de Mazunte. Finalmente, el Área Natural Protegida Santuario Playa de Escobilla, tiene una longitud de 15 kilómetros. y se localiza a unos 30 minutos en automóvil del Pueblo Mágico de Mazunte. El Instituto Nacional de Estadística y Geografía (INEGI, 2010 cit. en CONANP, 2018) refiere que, al interior del Santuario Playa de Escobilla no hay asentamientos humanos. Únicamente existen dos edificios: uno corresponde al campamento permanente ocupado por la Comisión Nacional de Áreas Naturales Protegidas (CONANP) y la

\footnotetext{
${ }^{1}$ De acuerdo con M. Hernández (oriunda de Mazunte desde finales de los años noventa y proveniente de un país de Europa), la llegada a la comunidad coincidió con un grupo de personas de distintos puntos del viejo continente y de Estados Unidos de América pertenecientes al movimiento hippie y New Age, quienes poco a poco encontraron en la localidad, un lugar de tranquilidad para residir. En palabras de la entrevistada, también hubo extranjeros provenientes de Canadá quienes compraron terrenos y edificaron inmuebles en Mazunte, pero que solo llegan en la temporada decembrina (M. Hernández, comunicación personal, 23 de mayo de 2019). Por otro lado, el Catálogo de Microrregiones de la Secretaría de Desarrollo Social (2013), establece que para 2010, la población de Mazunte era de 873 habitantes (434 hombres y 439 mujeres). Por su parte, Demetrio Pacheco, (Agente Municipal de Mazunte) sostiene que en 2019 alrededor de un 30\% de la población que habita la localidad son de origen extranjero. También, el funcionario refirió que, los países de origen de las y los extranjeros que habitan en Mazunte son variados: en una porción mayor son canadienses y estadounidenses, mientras existe una minoría de residentes de Europa y de América del Sur (D. Pacheco, comunicación personal, 18 de noviembre de 2019).

${ }^{2}$ El Catálogo de Microrregiones de la Secretaría de Desarrollo Social (2013), establece que para 2010, la población de Ventanilla era de 94 habitantes (43 hombres y 51 mujeres). Sin embargo, Alfonso Escamilla (Representante Comunitario de Ventanilla), refiere que, en 2019, la población de esta localidad oscilaba entre los 210 habitantes (A. Escamilla, comunicación personal, 25 de mayo de 2019).
} 


\section{Sección Especial - Territorio, Sustentabilidad, Cultura y Transformación de Espacios}

Secretaría de Marina (SEMAR). También, refiere que, en la zona circundante del santuario se ubican 11 localidades con 3,101 habitantes (p. 180).
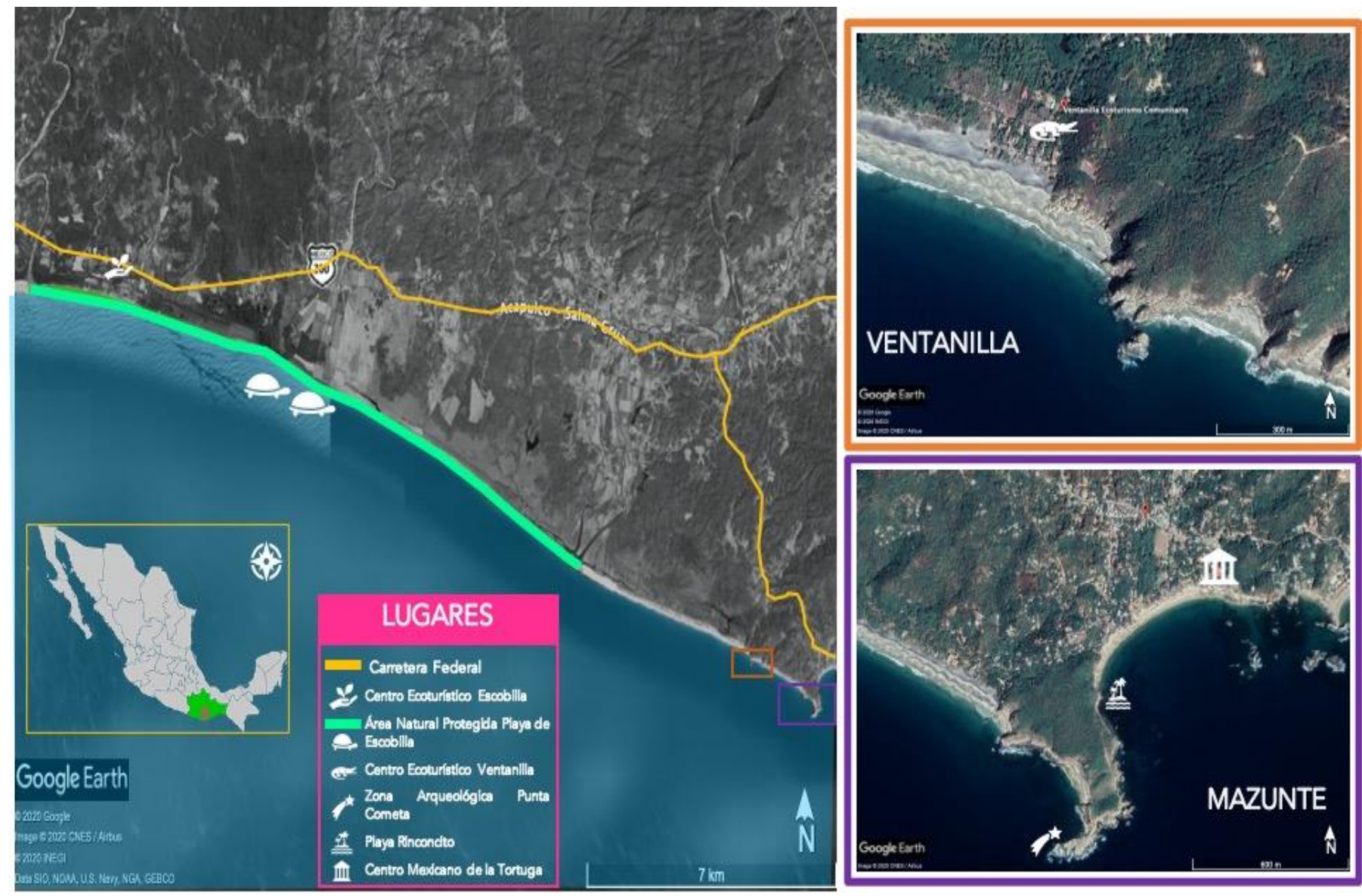

Figura 2. Localización del área de estudio: Corredor Mazunte- Escobilla, Oaxaca, México. Fuente: Elaboración propia a partir de Google Earth.

Los paisajes bioculturales que se aprecian en la zona son: zonas de playa, un santuario tortuguero, una zona de manglar, una Unidad de Manejo Ambiental, senderos interpretativos; así como la zona arqueológica Punta Cometa (Ver Figura 3). 


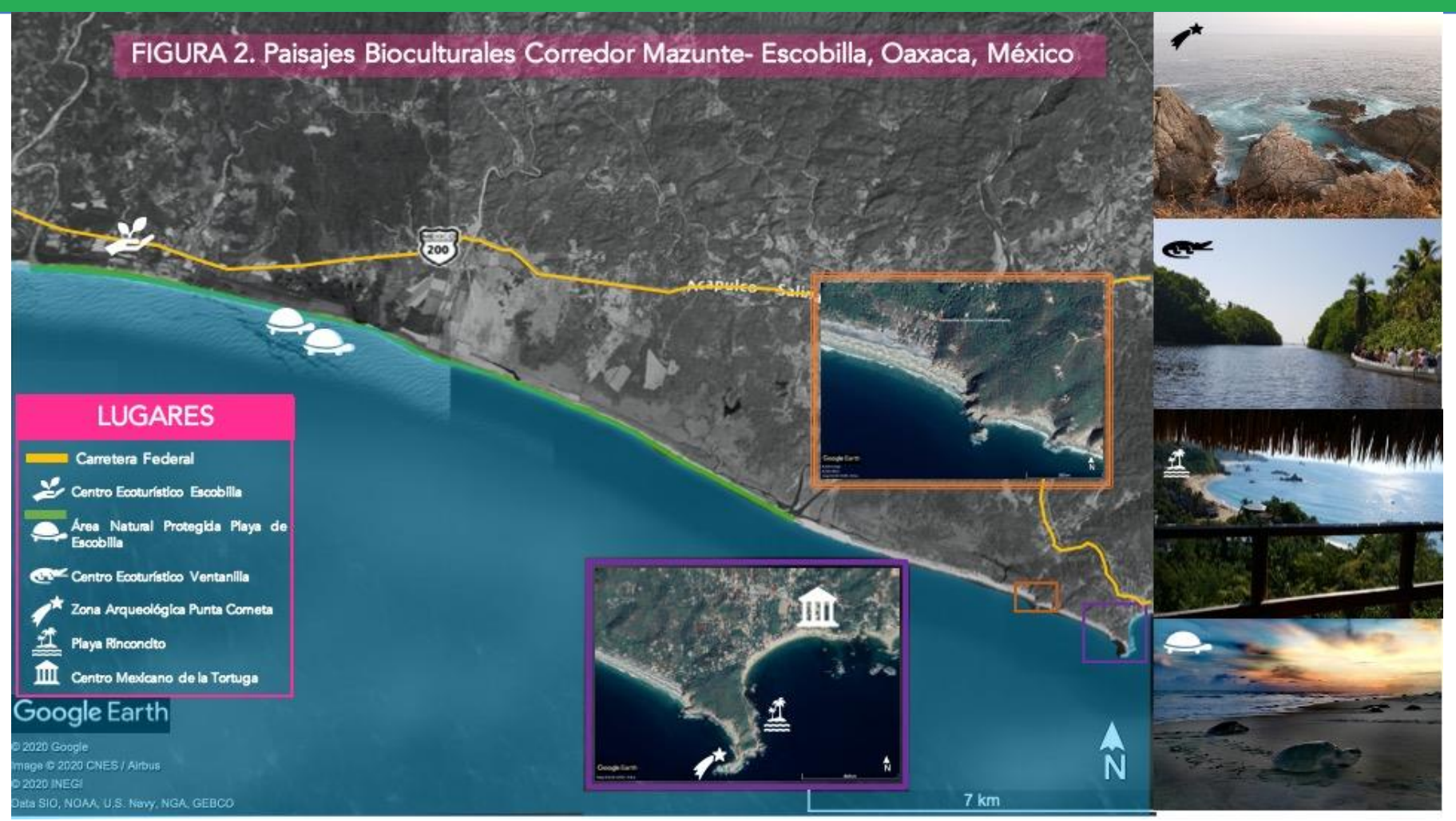

Figura 3. Paisajes Bioculturales Corredor Mazunte- Escobilla, Oax. Fuente: Elaboración propia a partir de Google Earth. Fotografías de arriba hacia abajo: 1) Punta Cometa; 2) Manglar de Ventanilla y; 3) Playa Rinconcito (Diana Zarate); 4) Playa Escobilla (@EdgarEnd).

\section{Movilización, disputa y ecoturismo}

Morales (2009), refiere que este territorio tuvo una primera etapa agrícola que abarcaba los años 1950 hasta 1967 en donde se desarrollaron una serie de conflictos sociales. Posteriormente, la actividad económica que más influencia generó fue a través de la pesca (1967-1990) y la de destazamiento de tortuga en Mazunte que se transportaban a un rastro ubicado en San Agustinillo, una playa cercana a Mazunte (págs. 135-141). Por su parte, Vargas del Río y Brenner (2013) refieren que, a diferencia de La Escobilla, los habitantes de Ventanilla no estuvieron adscritos a ninguna cooperativa y tampoco trabajaron como empleados del rastro, que desde 1967 existía en la comunidad cercana de Mazunte (p. 46). En lo que respecta a la comunidad de Ventanilla, Zamora (2009, cit. en Vargas del Río \& Brenner, 2013), relata que los pobladores de La Ventanilla solo se beneficiaron económicamente mediante la venta (ilegal) de huevos de tortuga marina que con frecuencia desovaba en su zona de la playa (p. 46). 


\section{Sección Especial - Territorio, Sustentabilidad, Cultura y Transformación de Espacios}

A finales del siglo XX, la región se comenzó a observar un detrimento del número de individuos de tortuga, lo cual decantó en el hecho de que autoridades ambientales federales configuraran un decreto para la prohibición de caza de tortuga. El decreto llevó a una movilización social pues la comunidad fue impedida para continuar con la pesca de tortuga, trayendo como consecuencia, una especie de despojo que concluyó en problemas de índole económico y social tales como la migración y la pobreza. Además, grupos ajenos a la comunidad llegaron bajo la premisa de que la conservación ambiental mediante el ecoturismo era una alternativa de desarrollo que permitiría una calidad de vida justa para la comunidad, especialmente la de Mazunte.

Al respecto, Gaudencio Cortés Martínez ${ }^{3}$, habitante de Mazunte sostiene lo siguiente:

En ese tiempo acá no se escuchaba mucho lo de los grupos ambientalistas. Ósea, una parte complicada porque los ambientalistas eran de la CDMX o de otras partes. Finalmente logran lo que quieren..., vedar la tortuga..., pero no hubo como muchas alternativas para la población. Esta zona, después de la veda de la tortuga, se cayó y mucha gente tuvo que migrar..., de aquí muchos jóvenes, muchas personas se tuvieron que ir hacia Estados Unidos. [...] Paralelamente empezó el proyecto de Huatulco, entonces algunas personas se fueron allá a participar en la construcción y muchos se fueron para allá. Aquí de pronto se vació. No había forma. También unas cooperativas cuando se viene lo de la veda, el gobierno si indemniza a las cooperativas y les dio lanchas, motores, partes de pesca, este..., pero a los trabajadores en sí no les tocó absolutamente nada. Por ejemplo, a los que trabajaban en el rastro..., mis hermanos, mis familiares, cuando se acabó, se acabó. Mazunte era una comunidad pequeñita. Estaba aislada. No había medios de transporte. Estaba solo la carreterita que llegaba a Puerto Ángel. En tiempo de cuaresma, se arreglaba. Pero de este lado de Mazunte, hasta San Antonio era peor. Por la parte del Zapotal era muy pantanoso. Como el 94 se veda. 95 y 96 son años muy complicados. En el 92 una "pre" veda temporal. Como del 91 al 95 todo el tiempo así. Alternativamente el gobierno federal, por las presiones que había sobre todo este asunto, este..., se viene lo del Centro Mexicano de la Tortuga. El gobierno lo traía a Huatulco [...], pero por presiones de las comunidades locales que se empezaron a organizar dijeron "pero cómo es posible que te lleves esto de las tortugas a Huatulco cuando lo de las tortugas estaban acá. Con algo nos tienen que compensar". Si hubo presión. También lo quería Puerto Ángel. También lo quería Escobilla, por ejemplo y finalmente se decide hacerlo acá.

Rosa (2002), refiere que, para el año de 1992, Mazunte se contaba con una Reserva Ecológica Campesina de 14,000 hectáreas y una asociación de comuneros que recibió la concesión del frente de playa. Posteriormente, "en 1999, Mazunte contaba con 400 camas, 12 restaurantes, un pequeño hotel, 30 negocios sobre la playa y cuatro taxis.

\footnotetext{
${ }^{3}$ G. Cortés, comunicación personal, 23 de mayo de 2019.

Recepción: 29/octubre/2019

Aceptación: 13/abril/2020

DOI: En asignación

UVserva 9 abril-septiembre 2020, ISSN: 2448-7430
}

167 
Sección Especial - Territorios, Sustentabilidad, Cultura y Transformación de Espacios

La mayor parte de la población vivía del turismo y existía una fábrica de cosméticos naturales y un Museo de la Tortuga (p. 3). En 1994, la Secretaría de Pesca, a través del Instituto Nacional de la Pesca, inició la operación del Centro Mexicano de la Tortuga (CMT), ubicado en la comunidad de Mazunte, Santa María Tonameca, a pocos kilómetros de la playa de Escobilla (CONANP, 2018, p. 17) para registrar el número de individuos y se preservara de alguna forma la especie.

Respecto a la construcción del Museo de la Tortuga, Mercedes Mendoza Ruíz ${ }^{4}$ señala lo siguiente:

De hecho, cuando quitaron lo que fue lo de la veda de las tortugas, mucha gente se desesperó y vendió sus terrenos a precios muy baratos..., el pueblo se quedó sin trabajo. Un tío que ya murió era hermano de mi papá él fue Agente Municipal del Pueblo varias ocasiones porque el pueblo lo quería mucho porque, no sé cómo le hacía, pero traía apoyo a la gente como la leche en polvo o de barras de queso amarillo. Acá se siembra el maíz y era más fácil hacerte unas quesadillas para comer. Entonces, se mencionó de un museo, entonces mi tío dijo pues vamos a pelear a que el museo se venga a Mazunte, entonces mi tío juntó gente del pueblo y fueron y pidieron el museo porque este museo no era para Mazunte, pero mi tío lo peleó y se vino a Mazunte. Cuando llegó este..., el museo aquí pues ya empezó a haber nuevo empleo de lo que fuera ¿no?, carpintería, albañilería, lo que fuera, le entró la gente porque mi tío lo inició este..., como que mi tío era el coordinador ahí y él dijo: "yo traje el museo para que la gente de Mazunte tenga trabajo, así que ahí le cayó todo Mazunte al trabajo y ahí tenían donde se hacían los acuarios. Obviamente vinieron arquitectos, gente preparada que tenía que hacerlo, pero pues los de Mazunte estuvieron ahí trabajando. Entonces empezaron a venir uno que otro turista y ahí entró un grupo de ECOSOLAR que nos decían que nos traían turistas y nosotros les dábamos la comida..., y así empezamos.

No obstante, del triunfo obtenido por la comunidad de Mazunte por llevar el Museo de la Tortuga a la localidad, el gobierno de ese entonces encabezado por el Partido de la Revolución Institucional (PRI), promovió diversos proyectos de infraestructura que afectaron a la comunidad. En este sentido, se podría sustentar que la generación por la disputa desde lo público fue haciéndose más evidente.

Como señala Cefaï (2012), el "público" no es un organismo social o político, sino más bien una forma extraña de vida colectiva, que emerge alrededor de un problema, siendo al mismo tiempo parte de él. Fue lo que sucedió en el territorio (p. 4). Las movilizaciones por parte de la comunidad en Mazunte a finales del siglo XX propulsaron una forma de experiencia pública que permitió la apropiación de espacios de poder, los cuales permitieron negociaciones frente

\footnotetext{
${ }^{4}$ M. Mendoza, comunicación personal, 22 de mayo de 2019.
} 


\section{Sección Especial - Territorio, Sustentabilidad, Cultura y Transformación de Espacios}

a los poderes políticos, económicos y administrativos del Estado de Oaxaca y del propio Gobierno Federal que después fue replicándose en Ventanilla y Escobilla. También, propulsó un fortalecimiento al Estado de Derecho de los paisajes bioculturales en esta región desde inicios del siglo XXI.

\section{Resultados}

Como primer hallazgo, se observa que el esquema de conservación presente en el corredor MAZ-ES, es el resultado de movilizaciones, conflictos, encuentros, desencuentros y negociaciones con las autoridades federales, estatales y locales. En la actualidad, el corredor MAZ-ES, cuenta con las siguientes cooperativas enfocadas a diversos servicios ecoturísticos:

1. Red de los Humedales de la Costa de Oaxaca (17 de agosto de 1989).

2. Cosméticos Naturales Mazunte (1994).

3. Cooperativa de Servicios Ecoturísticos La Ventanilla (CSELV) (1995).

4. Casa Mermejita (1998).

5. Cabañas Miramar (2002).

6. Cooperativa Lagarto Real (2004).

7. Sociedad Cooperativa el Santuario de la Tortuga La Escobilla S.C. de R.L. de C.V. (30 de agosto de 2004).

8. Cabañas el Copal Mazunte (2006).

De estas, se puede decir que la forma de enunciar y reconocer el ecoturismo es de forma solidaria

Otro hallazgo tiene que ver con el hecho de que, al interior del corredor MAZ-ES, existen diversos ecoturismos que no corresponden propiamente al ecoturismo solidario de las ocho cooperativas anteriormente citadas. Esto hace repensar el papel que puede tener la comunidad ante un escenario de despojo suave que pudiese propagarse en el territorio mediante la privatización del espacio comunitario reflejado en un mayor número de residentes del exterior ya sean nacionales y/o extranjeros y con ello, una forma de transculturación y hegemonización de la cultura local que pueda traer como consecuencia, la privatización de los paisajes bioculturales existentes en el corredor MAZ-ES.

Un último hallazgo versa en el hecho de que, la movilización social trajo una negociación con el gobierno federal y un "momento de crecimiento en términos económicos" para la comunidad. Sin embargo, la reorganización económica sobre la base de un ecoturismo parcial 
Sección Especial - Territorios, Sustentabilidad, Cultura y Transformación de Espacios

o capitalista del presente está transformando la visión y las experiencias de conservación de los paisajes bioculturales sobre todo en Mazunte. Esto, posiblemente se explique dada su denominación como Pueblo Mágico y su amplia difusión en tours y paquetes turísticos ofrecidos por tour operadoras o agentes de viajes que han consolidado un negocio respecto al valor del paisaje biocultural del territorio que ha traído como consecuencia problemas de estrés hídrico, inseguridad y competencia desleal en el costo de alimentos, bebidas y hospedaje.

En contraste con Mazunte, la comunidad de Ventanilla aún posibilita una experiencia más sensorial y biocultural con sus paisajes mediante los senderos interpretativos, su Unidad de Manejo Ambiental; así como con su Museo Comunitario. También, en el ANP Santuario Playa de Escobilla, la experiencia de observar las "arribadas" y liberación de tortugas supera, por mucho, el valor económico respecto al valor de uso en el disfrute de paisajes bioculturales excepcionales.

\section{Conclusiones}

Regular experiencias y no permitir la sobreexplotación de la naturaleza a través de la organización comunitaria organizada y crítica, sería la única forma de reestablecer la vida comunitaria y el equilibrio del valor del capital con relación al valor inmaterial de los paisajes bioculturales de la comunidad en el largo plazo. Educar en el conocimiento de los valores del territorio, como "mágicos" en el sentido no turístico, sino de vida, de equilibrio entre sus moradores y la naturaleza, puede ser también una manera de proteger esos ecosistemas. Educar para la vida, esa también debe ser una clave para el éxito.

\section{Referencias}

Barrera-Bassols, N. y Toledo, V. (2018). Devastación del Patrimonio Biocultural de México. Universidad Nacional Autónoma de México (Proyecto PAPIME: PE404318), en coedición con la Red para el Patrimonio Biocultural, CONACyT. Recuperado de: https://bit.ly/3awPqsD

Bauman, Z. (1998). La Globalización. Consecuencias humanas / Zygmunt Bauman; trad. De Daniel Zadunaisky. —2a ed. - México: FCE, 2001

Baumhackl, G. (2003). Ecoturismo y desarrollo sustentable en Mazunte, Oaxaca, México. Revista Ciencia y Mar. (7) 20. http://www.umar.mx/revistas/20/mazunte.pdf. 


\section{Sección Especial - Territorio, Sustentabilidad, Cultura y Transformación de Espacios}

Bezaury-Creel, J. \& Graf-Montero, S. \& Barclay-Briseño, K. \& Maza-Hernández, R. \& MachadoMacías, S. \& Sobral, E. \& Castilla, S. \& Ruíz-Barranco, H. (2015). Los Paisajes Bioculturales: un instrumento para el desarrollo rural y la conservación del patrimonio natural y cultural de México. DOI: 10.13140/RG.2.1.1420.9687.

Cefaï, D. (2012). ¿Qué es una arena pública? Algunas pautas para un acercamiento pragmático. http://lemetro.ifcs.ufrj.br/Cefai_Que_es_una_arena_publica_traduccion-libre.pdf

Cefaï, D. (2013) ¿Qué es la etnografía? Debates contemporáneos. Arraigamientos, operaciones y experiencias del trabajo de campo. Revista In Persona y Sociedad, 2013, XXVII, 1, pp. 101-119. Recuperado de: https://bit.ly/2yznbMK.

Comisión Nacional de Áreas Naturales Protegidas, CONANP (2018). Estudio Previo Justificativo para la modificación de la declaratoria de Santuarios de Playas Tortugueras. Recuperado de: https://bit.ly/2zqwlpJ.

Coordinación de Asesores de la Secretaría de Turismo (2018). TURISMO 2040. Política Turística de Estado. 5 Objetivos de Política Pública. Recuperado de: https://bit.ly/2S19oFj

Luque, D. \& Martínez-Y., \& Búrquez, A. \& López, G. \& Murphy, A. (2018). Los complejos Bioculturales; en Tópicos Bioculturales: reflexiones sobre el concepto de bioculturalidad y la defensa del patrimonio biocultural de México. Toledo, Víctor \& Alarcón-Cháires, Pablo (Editores). Recuperado de: https://bit.ly/2x2vU9L

Mardones, R. y Zunino, H. (2019). Repensando lo comunitario: discursos de comunidades internacionales utópicas en Chile. Convergencia. Revista de Ciencias Sociales e-ISSN 2448-5799, UAEM, 81, septiembre-diciembre 2019, pp. 1-21.https://bit.ly/2VUAZJo

Morales, M. (2009). Turismo y tenencia de la tierra en la costa de Oaxaca: Los casos de Mazunte y San Agustinillo. Tesis Licenciatura. Antropología Cultural. Departamento de Antropología, Escuela de Ciencias Sociales, Universidad de las Américas Puebla. Recuperado de: https://bit.ly/2VRkzBH

Obombo K. y Velarde M. (2019). El ecoturismo en las reservas de la biósfera: Prácticas y actitudes hacia la conservación. Pasos. Revista de Turismo y Patrimonio Cultural 17 (1), 97-112. https://doi.org/10.25145/j.pasos.2019.17.007

Ortiz, D. (2018). La elite local y su apropiación del Programa Pueblos Mágicos. URBS. Revista de Estudios Urbanos y Ciencias Sociales, 8(2), 107-120. Recuperado de: http://hdl.handle.net/10835/6364

Pérez, J. (2017). Urbanización y conflictos ambientales en suelo de conservación de Xochimilco, Ciudad de México. Revista Crecer Empresarial: Journal of Management and Development1(1), 61-73. Recuperado de: https://bit.ly/2xJjIv2

Rojas, A. y Palafox, A. (2018). La apropiación social del territorio en la Reserva de la Biosfera Sian Ka'an. Études caribéennes 41. 1-21

https://doi.org/10.4000/etudescaribeennes.13085 
Sección Especial - Territorios, Sustentabilidad, Cultura y Transformación de Espacios

Rosa, H. (Ed.) (2002). Pago por servicios ambientales y comunidades rurales: contexto, experiencias y lecciones de México. Informe elaborado en el marco del proyecto "Pago por Servicios Ambientales en las Américas" coordinado por PRISMA y patrocinado por la Fundación Ford. Recuperado de: https://bit.ly/2KwTmig

Secretaría de Desarrollo Social (2013). Catálogo de Localidades. Mazunte, Oaxaca, México. Sistema de Apoyo para la planeación de PDZP. Recuperado de: https://bit.ly/2Kv5yQU

Secretaría de Desarrollo Social (2013). Catálogo de Localidades. Ventanilla, Oaxaca, México. Sistema de Apoyo para la planeación de PDZP. Recuperado de: https://bit.ly/3bx4AQ1

Vargas del Río, D., \& Brenner, L. (2013). Ecoturismo comunitario y conservación ambiental: la experiencia de La Ventanilla, Oaxaca, México. Estudios sociales (Hermosillo, Son.), 21(41), 31-63. Recuperado de: https://bit.ly/351rc8U

Zarate, D. (octubre de 2019). ¿Qué es el Patrimonio Biocultural y cómo conservarlo? En G. Osorio (Presidenta) Foro "Hacia una Legislación para la Identificación y Protección del Patrimonio Biocultural de la Ciudad de México. Comisión de Derechos Culturales del Congreso de la Ciudad de México. 1a Legislatura. Foro llevado a cabo en el Lienzo Charro del Pedregal, Alcaldía Tlalpan, Ciudad de México 